\title{
LAS ACTITUDES DE LOS DOCENTES HACIA LA FORMACIÓN EN TECNOLOGÍAS DE LA INFORMACIÓN Y COMUNICACIÓN (TIC) APLICADAS A LA EDUCACIÓN.
}

\author{
Francisco D. Fernández Martín \\ Francisco Javier Hinojo Lucena \\ Inmaculada Aznar Díaz \\ Universidad de Granada
}

\begin{abstract}
RESUMEN. El propósito general del presente estudio es investigar las actitudes que los docentes y futuros docente poseen respecto a la formación en Tecnologías de la Información y la Comunicación aplicadas a la educación. Para ello, los sujetos participantes fueron elegidos aleatoriamente de centros de enseñanza, tanto rurales como urbanos, de la Provincia de Granada, así como de la Facultad de Ciencias de la Educación de la Universidad de Granada. Sus actitudes hacia la formación en TIC fueron evaluadas a través de una Escala Lickert de elaboración propia. Los resultados del estudio, de carácter descriptivo, nos dan a conocer, dentro de la formación y perfeccionamiento en TIC, las actitudes hacia las siguientes dimensiones: la aplicabilidad de las TIC en las diferentes áreas del currículum de Educación Primaria, la importancia de la formación en TIC aplicadas a la educación, el nivel y disponibilidad para la formación en TIC aplicadas a la educación, la formación inicial recibida en TIC aplicadas a la educación y la Formación permanente en TIC aplicadas a la educación.
\end{abstract}

ABSTRACT. The main purpose of this study is to research on teachers and future teachers' acttitudes towards their formation in Technologies of Information and Communication applied to Education. Therefore participants where chosen randomly in educational centers, rural, as well as urban ones, in the province of Granada, and the Faculty of Education of Granada.

Their attitudes towards the teaching of TIC where assessed thorough a self produced Likert Scale. The results of this study, descriptional results, show the attitudes found in different dimensions: The functionality of TIC in the Primary Education Curriculum, the need of teaching of TIC applied to education, the possibilities of studying TIC in Education y the Continual Learning of TIC applied to education.

\section{Introducción}

En las últimas décadas hemos podido observar un aumento tanto cualitativo como cuantitativo de las Tecnologías de la Información y Comunicación (TIC) en nuestra sociedad, lo que está dando lugar a una transformación de ésta. Ante esta situación, 
la escuela no puede dejar de lado las nuevas tecnologías de la información y comunicación, sino que debe preparar a las nuevas generaciones para convivir con estos medios promoviendo la participación y la reflexión crítica en su uso e interpretación, lo que a su vez, ha conllevado a la aparición de un gran reto: " La Formación del Profesorado en Tecnologías de la Información y la Comunicación".

A lo largo de la historia, podemos observar que han sido muchos los autores que han llevado a cabo investigaciones cuya finalidad era conocer las actitudes de los individuos en relación a diversos aspectos. Los trabajos pioneros en esta materia, fueron los realizado por Thurstone (1928), quién desarrollo diversas escalas con la finalidad de situar a los individuos en un continuo psicológico de afecto. Aún así, uno de los personajes más conocidos en este realidad fue Lickert (1932), quién diseñó uno de los métodos que más ha influido en este tipo de investigaciones. Concretando un poco más con nuestro trabajo, ya que sería imposible expresar todas las investigaciones que en esta línea se han llevado a cabo, hemos de decir que se han desarrollado investigaciones en las que se ha intentado conocer las actitudes de los profesionales y futuros profesionales de la educación hacia las Tecnologías de la Información y la Comunicación, pero no específicamente a la formación en TIC.

El propósito general del presente estudio es investigar las actitudes que los docentes y futuros docente poseen respecto a la formación en Nuevas Tecnologías de la Información y la Comunicación aplicadas a la educación. Para ello, los sujetos participantes fueron elegidos aleatoriamente de centros de enseñanza, tanto rurales como urbanos, de la Provincia de Granada, así como de la Facultad de Ciencias de la Educación de la Universidad de Granada. Sus actitudes hacia la formación en TIC fueron tomadas a través de una Escala Lickert que hemos elaborado nosotros mismos, ya que se adolece dentro de las producciones científicas de una escala cuyo objetivo de evaluación concuerde con la finalidad o propósito de esta investigación.

\section{Fundamentación Teórica}

\subsection{Las actitudes}

El concepto de actitud, como expresan Nieto y Sierra (1997: 57), "es un constructo que nos permite conocer las consistencia de los que las personas dicen, piensan o hacen, de forma que dadas determinadas conductas se pueden predecir otras futuras". A pesar de esto, podemos encontrar una gran variedad de definiciones debido a que las actitudes son un tema de estudio bastante controvertido. Así por ejemplo, Thurstone (1932), define la actitud como "la intensidad de afecto a favor o en contra de un objeto psicológico". Por su parte, Allport (1935), la define como "una disposición metal o neurológica de preparación para la acción que se organiza para la experiencia, y que ejerce una influencia directa o dinámica sobre la respuesta del individuo a todos los objetos y a todas las situaciones con las que se relaciona.

No obstante, la mayoría de las definiciones de actitudes, se pueden agrupar en tres categorías (Olson y Zanna, 1993): componente afectivo (sentimientos negativos hacia...), componente conductual (acciones o conductas negativas hacia...) y componente cognitivo (pensamientos negativos hacia...). 
Si como hemos observado, hay una falta de consenso a la hora de definir esta realidad, también para su estudio hay una gran cantidad escalas: escalas nominales, ordinales, de intervalo y de razón...

En cuanto a los métodos clásicos de construcción de escalas de actitudes, los más conocidos y utilizados son: la escala de distancia social, la escala Thurstone, la escala Guttman, el diferencial semántico y la escala Likert, que ha sido la que hemos elegido para realizar este estudio.

\section{La Formación Del Profesorado en nuevas tecnologías de la información y comunicación.}

\subsection{La formación inicial del profesorado en NTIC}

Actualmente, la formación en TIC durante la formación inicial de los docentes queda reducida a una sola materia: "Las Nuevas Tecnologías Aplicadas a la Educación", estrechamente ligada a las consecuencias sociales que están teniendo las tecnologías de la información y de la comunicación. También hemos de reconocer y tener en cuenta que en otras materias también se trabajan las TIC aplicadas a la educación ya que en la implementación de esa materia durante la práctica profesional se van a utilizar como recursos.

Centrándonos un poco más en esta disciplina, en el caso de la Universidad de Granada, las titulaciones de Magisterio (Educación Infantil, Educación Primaria, Educación Musical, Lenguas Extranjeras, Educación Física, Audición y Lenguaje y Educación Especial) contemplan en sus planes de estudio las Nuevas Tecnologías Aplicadas a la Educación como asignatura troncal, la cual se imparte en el último curso de la carrera (tercero), con una consideración de cuatro créditos y medio, de los cuales tres corresponden a una formación teórica y uno y medio son de carácter práctico.

A través de esta nueva disciplina el aspirante a maestro o futuro docente es iniciado en el estudio, aplicación, e integración curricular, desde una perspectiva crítica y reflexiva, de las nuevas tecnologías de la información y la comunicación, a través de una formación orientada a la práctica. A su vez, se pretende ofrecer unos instrumentos de tratamiento de la información y la comunicación a los docentes, para conseguir una mejora de la calidad de la enseñanza y un acercamiento a la realidad social. También se pretende que adquieran una capacidad de analizar las implicaciones pedagógicas y las posibilidades reales de innovación que permiten estos nuevos recursos didácticos.

\subsection{La Formación y perfeccionamiento del Profesorado en TIC}

2.2.1. La falta de capacitación en TIC de los profesionales de la enseñanza

De forma similar a como están influyendo las TIC en la sociedad, alterando los criterios y principios que sobre la comunicación se han venido manteniendo a lo largo del tiempo, podemos deducir que sus repercusiones afectan a todas las estructuras sociales y entre ellas al Sistema Educativo. 
Cuando se introduce un cambio como éste en la sociedad, la escuela como reflejo de ella tiende también a cambiar, pero esto no conlleva una oposición al cambio de los miembros de la comunidad educativa, como se podría pensar o como sucedería en otros ámbitos, todo lo contrario, aquello que facilite o de mayor calidad al proceso de E-A es bienvenido. Las dificultades o problemas aparecen cuando el profesorado no se siente formado en ese ámbito, es decir, en la aplicación de las TIC en la educación.

Según las investigaciones en este campo, la mayoría de las veces este problema de utilización de las TIC como recurso dentro del proceso de E-A o dentro del campo educativo en general, se debe a una o varias de las siguientes causas:

- Falta de presencia de las TIC en los centros, por falta de recursos.

- Limitada formación del profesorado para su utilización.

- Actitudes de desconfianza y temor hacia las TIC por parte de los profesores.

- El conocimiento limitado teórico y práctico respecto a cómo funcionan las TIC en el contexto educativo.

- El tradicionalismo en el que tiende a desenvolverse la escuela.

- La falta de ofertas formativas sobre TIC y tendencia de éstas a una capacitación instrumental.

- Costo de adquisición y mantenimiento de los equipos.

- El asentamiento en el trabajo, que conlleva una pasividad del profesor.

- Falta de tiempo y capacitación del profesorado para producir sus propios materiales de enseñanza.

- Estructura organizativa de los centros educativos.

- La falta de estudios y/o investigaciones al respecto.

Pese a todos estos inconvenientes, del que destaca la falta de formación de los docentes junto con la ausencia de TIC en los centros, urge la necesidad de formar a los profesionales de la enseñanza en TIC ya que en la actualidad el aprendizaje de nuestros alumnos está dominado por la tecnología de la que disponen en sus hogares o a la que acceden en otros lugares y que usan de un modo acrítico e irreflexivo.

Medina (1989) señala tres aspectos fundamentales que justificarían la necesidad de una formación del profesorado en este ámbito:

a) Mejorar su interpretación y concepción tecnológica de la enseñanza desde el protagonismo reflexivo del profesor como generador de currículum y estilos de enseñanza.

b) Alcanzar una concepción tecnológica apoyada en una fundamentación científica del proceso enseñanza-aprendizaje y en la actualización artístico-reflexiva en el aula.

c) Gestionar y organizar los medios en el aula y en el centro. Aquí habría que entrar en dinámicas de colaboración y reparto de responsabilidades en equipos de profesores. 
2.2.2. Propuestas para abordar la formación y perfeccionamiento del profesorado en TIC

Muchos han sido los autores que han tratado las formas desde las que abordar la formación y el perfeccionamiento del profesorado en Tecnologías de la Información y la Comunicación.

Blázquez (1994) nos habla de los propósitos formativos que se deben alcanzar dentro de la formación de los maestros en nuevas tecnologías de la información y comunicación: 1) despertar un sentido crítico hacia los medios, 2) relativizar el no tan inmenso poder de los medios, 3) analizar el contenido de los medios tanto su empleo como expresión creadora, 4) conocer los sustratos ocultos de los medios, 5) conocer las directrices españolas o europeas sobre los medios, 6) conocimiento y uso en el aula de los denominados medios audiovisuales, 7) investigación sobre los medios, 8) pautas para convertir en conocimientos sistemáticos los saberes desorganizados que los niños y los jóvenes obtienen de los mass-media, 9) un mínimo conocimiento técnico, y 10) reflexionar sobre las consecuencias en la enseñanza de los nuevos canales tanto organizativas como sobre los contenidos y las metodologías.

Por su parte, Alonso y Gallego (1996) plantean que los docentes de hoy en día deben de desempeñar quince funciones básicas, de las cuales se desprenden propuestas para su formación y perfeccionamiento. Estas funciones son: 1) favorecer el principal objetivo: el aprendizaje de los alumnos, 2) utilizar los recursos psicológicos del aprendizaje, 3) estar predispuestos a la innovación, 4) poseer una actitud positiva ante la integración de nuevos medios tecnológicos en el proceso de enseñanza-aprendizaje, 5) integrar los medios tecnológicos como un elemento más del diseño curricular, 6) aplicar los medios didácticamente, 7) aprovechar el valor de comunicación de los medios para favorecer la transmisión de información, 8) conocer y utilizar los lenguajes y códigos semánticos, 9) adoptar una postura crítica, de análisis y de adaptación al contexto escolar, de los medios de comunicación, 10) valorar la tecnología por encima de la técnica, 11) poseer las destrezas técnicas necesarias, 12), diseñar y producir medios tecnológicos, 13) seleccionar y evaluar los recursos tecnológicos, 14) organizar los medios, y 15) investigar con y sobre medios.

Por otro lado, Cebrián de la Serna (1996) nos sugiere que esta formación debe de perseguir cinco objetivos básicos:

1. Los procesos de comunicación y de significación que generan las distintas nuevas tecnologías.

2. Las diferentes formas de trabajar las nuevas tecnologías en las distintas disciplinas y áreas.

3. Los conocimientos organizativos y didácticos sobre el uso de las nuevas tecnologías en la planificación del aula.

4. Los conocimientos organizativos y didácticos sobre el uso de las nuevas tecnologías en la planificación del aula y del centro y organización de los recursos en los planes de centros como en la programación del aula.

5. Y los criterios válidos para la selección de materiales, así como conocimientos técnicos suficiente para permitirle rehacer y estructurar de nuevo los materiales existentes en el mercado para adaptarlo a sus necesidades, como crear otras totalmente nuevas. 
Por último, podemos comentar la propuesta de Ballesta (1996), quien nos habla que la formación y perfeccionamiento del profesorado en TIC debe pretender alcanzar una serie de descriptores, como los siguientes:

1. Formación para el uso crítico de las nuevas tecnologías.

2. Desarrollar la motivación en el usuario.

3. Aprendizaje de situaciones reales.

4. Diseño de modelos de experimentación.

5. Realización de propuestas didácticas en el aula.

6. Ampliación de tratamientos interdisciplinares.

Y colaboración de centros educativos y empresas comunicativas.

Como hemos podido observar, no estamos ante una empresa fácil. La formación de los docentes en TIC aplicadas a la educación es necesaria e importantísima, tanto para los alumnos como para los mismo profesionales de la educación. Ante esta situación, se hacen necesarios estudios de naturaleza similar o parecida a éste, con la finalidad de conocer las actitudes y barreras o posibles barreras que nos encontramos a la hora de abordar la formación y perfeccionamiento en TIC aplicadas a la educación. Pero esto no puede quedar aquí, sino que se ha de ir más allá, es decir, debemos poner en marcha todos los medios necesarios para cambiar esas actitudes (nuestras conductas se materializan en función de nuestras actitudes) y superar esas barreras.

\section{Objetivos del estudio}

El objetivo del presente estudio es investigar las actitudes que los docentes y futuros docente poseen respecto a la formación en Tecnologías de la Información y la Comunicación aplicadas a la educación. Sus actitudes hacia la formación en TIC fueron evaluadas a través de una Escala tipo Lickert de elaboración propia.

Los propósitos específicos del estudio fueron:

- Conocer las actitudes de los docentes y futuros docentes hacia la aplicabilidad de las TIC en las diferentes áreas del currículum de Educación Primaria.

- Conocer las actitudes de los docentes y futuros docentes hacia la importancia de la formación en TIC aplicadas a la educación.

- Conocer las actitudes de los docentes y futuros docentes hacia el nivel y disponibilidad para la formación en TIC aplicadas a la educación.

- Conocer las actitudes de los docentes y futuros docentes hacia la formación inicial recibida en TIC aplicadas a la educación.

- Conocer las actitudes de los docentes y futuros docentes hacia la Formación permanente en TIC aplicadas a la educación.

\section{Método}

Muestra

La muestra ha estado compuesta por 241 sujetos de la provincia de Granada seleccionados aleatoriamente, de los cuales: 
- 77 son profesores en zonas urbanas,

- 79 son profesores en zonas rurales

- 85 son futuros profesores, es decir, profesores en formación de la Facultad de Ciencias de la Educación de la Universidad de Granada.

En cuanto a la división de la muestra por sexos, 119 eran mujeres y 122 varones. La Edad de la muestra oscila entre 19 y 53 años, con una media de 32.66. Todo esto los podemos observar más detalladamente en la Tabla 1.

\section{Instrumento}

El instrumento utilizado para realizar el estudio ha sido una escala de actitudes tipo Likert de elaboración propia, en el que a través de 20 preguntas o ítem con una posibilidad de respuesta de entre 1 y 5 (1: Totalmente en desacuerdo; 2: En desacuerdo; 3: Ni de acuerdo ni en desacuerdo; 4: En acuerdo; 5: Totalmente de acuerdo), se intenta hacer un recorrido por aquellos aspectos que conforman el marco actitudinal del profesorado en relación a la formación y perfeccionamiento en Nuevas Tecnologías de la Información y Comunicación.

El procedimiento de construcción, paso a paso, que hemos seguido para elaborar la Escala fue el siguiente:

$1^{\text {a }}$ Fase) Elección de un objeto actitudinal (la formación y perfeccionamiento en NTIC).

$2^{2}$ Fase) Elaboración de los ítem iniciales.

$3^{\text {a }}$ Fase) Construcción de una escala piloto.

$4^{\text {a }}$ Fase) Administración de la escala a un grupo de sujetos.

$5^{\text {a }}$ Fase) Construcción de una matriz de datos.

$6^{\text {a }}$ Fase) Eliminación de sujetos con puntuaciones intermedias en la escala.

$7^{\text {ạ }}$ Fase) Análisis y selección de los ítem.

$8^{\text {a }}$ Fase) Balanceado y determinación de la escala definitiva.

9a Fase) Escala Actitudinal Definitiva, que ha sido la utilizada en este estudio.

Esta escala tiene la finalidad de darnos a conocer las actitudes que los docentes y futuros docente tienen con respecto a:

- La aplicabilidad de las TIC en las diferentes áreas y estudios.

- La importancia de la Formación en TIC.

- La disponibilidad hacia la formación en TIC.

- La formación Inicial en TIC.

- La formación Permanente TIC.

\section{Análisis de datos}

Para llevar a cabo el análisis estadístico de los datos fue utilizado el Paquete Estadístico de las Ciencias Sociales (SPSS). Este análisis se limitó, debido a la naturaleza del estudio, a la realización de la estadística descriptiva del mismo. 


\section{Resultados}

Los resultados del estudio, que a continuación pasaremos a desglosar, son de carácter descriptivo, por lo que para facilitar su interpretación los hemos descrito en tantos por ciento; los valores en \% que aparecen en el texto son aproximativos, por lo que para una mayor precisión de los datos se han de observar las Tablas.

Aplicabilidad de las NTIC en las diferentes áreas

Como observamos en la tabla 2, las actitudes de los docentes y futuros docentes hacia la aplicabilidad de las TIC a las diferentes áreas del currículum de educación Primaria, podemos expresarla de la siguiente forma:

- Educación Física: un 64\% de los participantes piensa que las TIC son aplicables a esta área, mientras que un $10 \%$ opina todo lo contrario, quedando un $25 \%$ en una posición neutra.

- Educación Musical: un 69\% de los participantes piensa que las TIC son aplicables a esta área, mientras que un $12 \%$ opina todo lo contrario, quedando un $19 \%$ en una posición neutra.

- Educación Artística: un 78\% de los participantes piensa que las TIC son aplicables a esta área, mientras que un $10 \%$ opina todo lo contrario, quedando un $12 \%$ en una posición neutra.

- Matemáticas: un $86 \%$ de los participantes piensa que las TIC son aplicables a esta área, mientras que un $11 \%$ opina todo lo contrario, quedando un $2 \%$ en una posición neutra.

- Lengua y Literatura: un $87 \%$ de los participantes piensa que las TIC son aplicables a esta área, mientras que un $6 \%$ opina todo lo contrario, quedando un $2 \%$ en una posición neutra.

- Idioma Extranjero: un 91\% de los participantes piensa que las TIC son aplicables a esta área, mientras que un $6 \%$ opina todo lo contrario, quedando un $6 \%$ en una posición neutra.

- Conocimiento del Medio: un $81 \%$ de los participantes piensa que las TIC son aplicables a esta área, mientras que un $12 \%$ opina todo lo contrario, quedando un $6 \%$ en una posición neutra.

En cuanto a la compatibilidad de las TIC con las materias que imparten los docentes en los centros o con las que se están especializando para impartir, un 70\% aproximadamente opinan que las TIC compatibles mientras que un $24 \%$ manifiestan todo lo contrario, quedando un $4 \%$ en una posición neutra.

En relación a la confianza que depositan tanto docentes como futuros docentes en las TIC como recursos didácticos, un $67 \%$ confían en ellas como tal mientras que un $8 \%$ manifiestan todo lo contrario, quedando un $24 \%$ en una posición neutra.

Importancia de la formación en TIC

Como observamos en la tabla 3, aproximadamente un 95\% de los docentes y futuros docentes del estudio piensan que la formación en TIC es importante, en contra- 
posición con aproximadamente un $3.7 \%$ que piensa que no es así, quedando un $0.4 \%$ en una posición neutra.

A su vez, un $87 \%$ aproximadamente, piensa que también es importante una formación en nociones básicas, en contra de un $0.4 \%$ que opina todo lo contrario y quedando un $12 \%$ en una posición nula.

En cuanto a la motivación del uso de las TIC en el centro, fruto de una mayor formación en ellas, los participantes, en un $87 \%$ aproximadamente, están de acuerdo, mientras que en un $4 \%$ opinan todo lo contrario, quedando un $8 \%$ en una posición neutra.

En relación a si la formación en TIC mejora el desarrollo profesional, un 91\% aproximadamente de la muestra, opina que si, en contraposición a un $8 \%$ que opina que no.

A su vez, un elevado número de los participantes en el estudio, un $54 \%$ aproximadamente, manifiesta una gran ausencia de las TIC en sus centros de trabajo. Igualmente, un $22 \%$ pone de manifiesto que en su centro la presencia de TIC es baja.

Necesidad, nivel y disponibilidad para la formación en TIC

Como observamos en la Tabla 4, los participantes, en aproximadamente un $62 \%$, manifiestan una necesidad de formación en TIC, en contra de un $4.5 \%$ que creen no necesitar esa formación. En cuanto a la necesidad de actualizar la formación en TIC, un $77 \%$ aproximado de los participantes cree que es necesaria para ellos, mientras que un $6.2 \%$ opina todo lo contrario, quedando un $8 \%$ en una posición neutra.

En relación a la disponibilidad para formarse en $\mathrm{TIC}$, los docentes y futuros docentes plasman, en un $70 \%$ aproximadamente, una gran disponibilidad para formarse en TIC, en contra posición a un $6 \%$, que opina todo lo contrario, quedando un $12 \%$ en una posición neutra. Por otra parte, los participantes en un 50\% aproximadamente, estarían dispuestos a estudiar cualquier oferta de formación que llegara a sus manos y realizarla, mientras que en un $37 \%$ haría todo lo contrario, quedando un $12 \%$ en una posición neutra.

\section{Formación Inicial}

Como observamos en la Tabla 5, en relación a las diferentes dimensiones de la Formación inicial que han recibido los participantes en el estudio que hemos intentado conocer, podemos decir que:

- Un $72 \%$ piensa que es insuficiente, frente a un $4 \%$ que opina todo lo contrario, quedando un $23 \%$ en una posición neutra.

- Un $46 \%$ piensa que es demasiado instrumentalista, frente a $33 \%$ que piensa todo lo contrario, quedando un $12 \%$ en una posición neutra.

- Un 66\% introduciría más asignaturas relacionadas con las NTIC aplicadas a la educación, frente a un $20 \%$ que no las introduciría, quedando un $12 \%$ en una posición neutra.

\section{Formación permanente}

Como podemos observar en la Tabla 6, en relación a las dimensiones de la formación permanente que hemos intentado conocer de los participantes en el estudio, podemos decir que: 
- Un 39\% aproximado de la muestra manifiesta que la formación permanente tiene un coste demasiado elevado, mientras que $51 \%$ manifiesta que es normal y $8 \%$ que no es elevada.

- Un $50 \%$ de la muestra manifiesta que en el lugar donde desempeña su función docente hay ofertas de formación en $\mathrm{TIC}$, mientras que un 33\% carece de ellas, quedando un $16 \%$ en una posición neutra.

- Un $41 \%$ de los participantes en el estudio manifiesta que ha realizado cursos, jornadas... relacionadas con las TIC aplicadas a la educación, mientras que un 33\% manifiesta todo lo contrario, quedando un $24 \%$ en una posición neutra.

- Un 54\% de los componentes de la muestra expresa que no tiene tiempo para formarse en $\mathrm{TIC}$, mientras que un $37 \%$ expresa todo lo contrario, quedando un $8 \%$ en una posición neutra.

- Un 50\% de los participantes en el estudio manifiesta que los cursos de formación permanente son demasiado instrumentalistas, mientras que un $36 \%$ manifiesta todo lo contrario, quedando un $13 \%$ en una posición neutra.

\section{Discusión y conclusiones}

El objetivo del presente estudio fue investigar las actitudes de los docentes y futuros docentes respecto a la formación en Tecnologías de la Información y la Comunicación aplicadas a la educación. Como era de esperar, el objetivo que nos habíamos marcado con esta investigación, además de los objetivos específicos, han sido cubiertos ya que el estudio es de carácter descriptivo.

Para llegar a la conclusión final del estudio vamos a ir analizando paso a paso cada una de las variables. Podemos decir que actualmente los docentes y futuros docentes demuestran unas actitudes bastante positivas hacia la utilización de las TIC en el aula y la importancia de la formación para el uso didáctico de éstas. Como hemos podido observar en los resultados que hacen referencia a la aplicación de las TIC en las diferentes áreas del currículum, la gran mayoría de los docentes y futuros docentes creen que las TIC aplicadas a la educación pueden ser utilizadas para el desarrollo de las diferentes áreas del currículum, eso si en algunas más que otras. A su vez, la gran mayoría confían en ellas como medio didáctico. Por otra parte, consideran esencial la formación en TIC aplicadas a la educación con la finalidad de hacer un adecuado uso de ellas, aprovechando todas las ventajas y beneficios que puedan aportar al proceso de Enseñanza-Aprendizaje, y mejorando su desarrollo profesional como docentes. Además, la gran mayoría de los docentes percibe que necesita formarse en TIC aplicadas a la educación o actualizar su formación en éstas.

A partir de lo hasta aquí descrito, podemos llegar a algunas conclusiones. Los docentes creen que las TIC son aplicables a las diferentes áreas del currículum, piensan que es importantísima la formación en TIC aplicadas a la educación y creen que necesitan formarse o actualizar su formación en ellas. Así, una de las causas de la escasa utilización de las TIC en las aulas es la falta de formación, a pesar de que los profesionales de la enseñanza creen que es necesaria e importantísima, además de aplicable para desarrollar todas las áreas del currículum. Pero, si los docentes perci- 
ben esa necesidad de formación ¿por qué no la llevan a cabo?. Sigamos analizando, a partir de los resultados, otras variables que entran en juego.

Comencemos por la formación inicial, la gran mayoría de los docentes piensa que esta formación es insuficiente e instrumentalista, e incluso abogan por un mayor número de asignaturas relacionadas con las TIC aplicadas a la educación durante su formación inicial. Por tanto, podemos decir que esa falta o necesidad de formación, en parte, se debe a una mala formación inicial ya que debería de ser la encargada de disminuir tal necesidad. Además, se puede concluir que están motivados e interesados en recibir una formación inicial en TIC, pero que está sea de calidad, de ahí que en ocasiones se produzca un efecto rebote y un tanto de rechazo a utilizar estos medios en el aula. Si continuamos con la formación permanente, muchos de los docentes piensan que su coste es algo elevado, hay ausencias de ofertas de formación en este ámbito en el lugar donde desarrolla su profesión docente, son demasiado instrumentalistas, y además, el tiempo de que disponen para esta formación es mínimo. Todo esto nos lleva a pensar, por un lado, que la oferta formativa no cumple las necesidades de los docentes en activo, y por otro, que no hay una oferta formativa de calidad en algunos casos y otros, existe un total aislamiento por parte de la administración educativa en formar a estos profesionales en algunas zonas más alejadas de la capital. Este es un dato que se repite con frecuencia, y una posible solución sería la motivación permanente y el acercamiento de este tipo de medios a todas las zonas donde actualmente no llegan. Se constata, en alguna entrevista de forma informal, que en muchos casos los medios existen en el Centro Educativo, pero que en la mayoría de los casos, no existe profesorado capaz de ponerlo en funcionamiento y siguen llevando a cabo clases de corte tradicional, sin incorporar estas tecnologías a su práctica diaria.

Por tanto, podemos concluir que actualmente los docentes, poseen unas actitudes positivas hacia la formación en TIC, pudiendo utilizarlas para el desarrollo de las áreas curriculares que imparten o se están preparando para impartir como medio didáctico, pero lo que más incide en esa falta de formación en TIC aplicadas a la educación, y su consecuente ausencia en el aula, son otras variables, no tanto los docentes en sí como se había venido pensando. Dentro de estas variables podemos nombrar:

- Falta de tiempo para llevar a cabo su formación en TIC.

- Ausencia en el centro donde desempeñan su función docente de TIC.

- Pocas ofertas de formación en TIC en el contexto donde desarrollan su trabajo.

- Elevado coste demasiado instrumentalista en las ofertas de formación en TIC.

- Formación inicial y permanente que no cubren las necesidades actuales.

Como podemos observar, debemos centrarnos en minimizar o intervenir directamente en esta serie de variables que inciden negativamente en la formación de nuestros docentes en TIC aplicadas a la educación, con la finalidad máxima de que usen adecuadamente estos recursos en el proceso de enseñanza-aprendizaje ayudándoles a conseguir los objetivos que se hayan marcado. Por todo ello, debemos concienciar a los docentes que sigan formándose en este campo mediante otros canales que no sean los tradicionales (llegado el caso que no tengan acceso), por ejemplo la autoformación mediante cursos on line, o la simple indagación. Es por ello, que se pide una 
actitud más positiva y más activa, en definitiva, y no, en algunos casos como se ha comprobado, que esperan de una forma pasiva a que se lo pongan en bandeja. Quizás sea mucho por nuestra parte esperar esta actitud sin una previa acción motivadora por parte de la Administración Educativa.

Para futuras investigaciones en este campo, recomendaríamos que se aumentara el número de sujetos participantes en el estudio y se hicieran diferenciaciones entre los docentes rurales, docentes urbanos y futuros docentes para conocer la realidad de cada uno de ellos. También recomendaríamos que el número de ítems de la escala Lickert que hemos utilizado se aumentara, complementándola con otras dimensiones de la formación en TIC aplicadas a la educación. En nuestro caso, el número de ítems, ha sido mayor pero hemos tenido que simplificarlos por necesidades de espacio y por aterrizar mejor en el cumplimiento de los objetivos propuestos.

También debemos de manifestar que nuestro trabajo, como la gran mayoría, posee algunas limitaciones. Somos conscientes de ello. La propia naturaleza descriptiva del mismo es una de sus mayores limitaciones ya que podrían analizarse los datos desde otras dimensiones. También la muestra puede presentar ciertas limitaciones ya que no es demasiado grande, aunque si representativa. Y por último, podríamos hablar de la escala Lickert, que carece de cierto grado de fiabilidad y validez, pero cabe destacar, la rica información desprende la misma.

TABLA 1. Muestra del estudio.

\begin{tabular}{|l|c|c|c|c|c|}
\hline \multicolumn{7}{|c|}{ MUESTRA DEL ESTUDIO } \\
\hline & MUJERES & HOMBRES & TOTAL & EDAD & $\begin{array}{c}\text { MEDIA } \\
\text { EDAD }\end{array}$ \\
\hline $\begin{array}{l}\text { Profesores en } \\
\text { Zona Rural }\end{array}$ & 35 & 42 & 73 & $26-42$ & 34 \\
\hline $\begin{array}{l}\text { Profesores en } \\
\text { Zona Urbana }\end{array}$ & 40 & 39 & 79 & $35-51$ & 43 \\
\hline $\begin{array}{l}\text { Futuros } \\
\text { profesores }\end{array}$ & 42 & 43 & 85 & $19-23$ & 21 \\
\hline
\end{tabular}


LAS ACTITUDES DE LOS DOCENTES HACIA LA FORMACIÓN

TABLA 2. Aplicabilidad de las TIC a las diferentes áreas

\begin{tabular}{|l|c|c|c|c|c|}
\hline & $\begin{array}{l}\text { Totalmente en } \\
\text { desacuerdo }\end{array}$ & $\begin{array}{c}\text { En } \\
\text { desacuerdo }\end{array}$ & Neutro & $\begin{array}{c}\text { De } \\
\text { acuerdo }\end{array}$ & $\begin{array}{c}\text { Totalmente } \\
\text { de acuerdo }\end{array}$ \\
\hline E. Física & 4.149 & 6.564 & 25.047 & 31.195 & 33.045 \\
\hline E. Musical & 9.298 & 1.415 & 19.749 & 40.493 & 29.045 \\
\hline E. Artística & 5.150 & 4.564 & 12.447 & 40.495 & 37.344 \\
\hline Idioma Extr. & 6.149 & 0.414 & 2.150 & 48.643 & 42.644 \\
\hline Matemáticas & 6.414 & 5.149 & 2.297 & 46.643 & 39.497 \\
\hline $\begin{array}{l}\text { Lengua y } \\
\text { Literatura }\end{array}$ & 5.149 & 1.414 & 6.298 & 40.496 & 46.643 \\
\hline $\begin{array}{l}\text { Conocimiento } \\
\text { del Medio }\end{array}$ & 10.298 & 2.424 & 6.145 & 37.341 & 43.792 \\
\hline $\begin{array}{l}\text { Compatibles } \\
\text { con las } \\
\text { materias } \\
\text { que imparto }\end{array}$ & 8.298 & 16.597 & 4.149 & 33.197 & 37.759 \\
\hline $\begin{array}{l}\text { Desconfianza } \\
\text { como medio } \\
\text { didáctico }\end{array}$ & 54.358 & 12.548 & 24.796 & 4.049 & 4.249 \\
\hline
\end{tabular}

TABLA 3. Actitudes hacia la importancia de la formación en NTIC de los participantes de la muestra en $\%$.

\begin{tabular}{|l|c|c|c|c|c|}
\hline \multicolumn{1}{|l|}{$\begin{array}{l}\text { Totalmente de } \\
\text { acuerdo }\end{array}$} & $\begin{array}{c}\text { De } \\
\text { acuerdo }\end{array}$ & Neutro & $\begin{array}{c}\text { En } \\
\text { acuerdo }\end{array}$ & $\begin{array}{c}\text { Totalmente } \\
\text { en desacuerdo }\end{array}$ \\
\hline $\begin{array}{l}\text { Importancia de } \\
\text { la Formación } \\
\text { en NTIC }\end{array}$ & 74.688 & 20.746 & 0.808 & 3.414 & 0.344 \\
\hline $\begin{array}{l}\text { Importancia de } \\
\text { la Formación en } \\
\text { NTIC en } \\
\text { Nociones Básicas }\end{array}$ & 58.091 & 29.045 & 12.448 & 0.416 & 0 \\
\hline $\begin{array}{l}\text { La formación en } \\
\text { NTIC motiva el } \\
\text { uso de ellas }\end{array}$ & 45.643 & 41.493 & 8.715 & 0 & 4.149 \\
\hline $\begin{array}{l}\text { La Formación en } \\
\text { NTIC mejora el } \\
\text { desarrollo } \\
\text { profesional } \\
\text { docente }\end{array}$ & 49.792 & 41.493 & 0 & 1.659 & 7.056 \\
\hline $\begin{array}{l}\text { Ausencia en el } \\
\text { centro de NTIC }\end{array}$ & 29.463 & 25.045 & 22.746 & 8.298 & 14.448 \\
\hline
\end{tabular}


FRANCISCO D. FERNÁNDEZ MARTÍN, FRANCISCO JAVIER HINOJO LUCENA, INMACULADA AZNAR DÍAZ

TABLA 4. Actitudes hacia la necesidad, nivel y disponibilidad para la formación en TIC de los participantes de la muestra en $\%$.

\begin{tabular}{|l|c|c|c|c|c|}
\hline & $\begin{array}{l}\text { Totalmente en } \\
\text { desacuerdo }\end{array}$ & $\begin{array}{c}\text { En } \\
\text { desacuerdo }\end{array}$ & Neutro & $\begin{array}{c}\text { De } \\
\text { acuerdo }\end{array}$ & $\begin{array}{c}\text { Totalmente } \\
\text { de acuerdo }\end{array}$ \\
\hline $\begin{array}{l}\text { Necesidad } \\
\text { de formarse } \\
\text { en NTIC }\end{array}$ & 0.417 & 4.149 & 8.298 & 8.298 & 53.941 \\
\hline $\begin{array}{l}\text { Necesidad } \\
\text { de Actualizar } \\
\text { la formación } \\
\text { en NTIC }\end{array}$ & 0 & 6.299 & 16.597 & 41.909 & 35.195 \\
\hline $\begin{array}{l}\text { Disponibilidad } \\
\text { a formarse } \\
\text { en NTIC }\end{array}$ & 0 & 6.299 & 12.864 & 14.447 & 66.390 \\
\hline $\begin{array}{l}\text { Ofertas de } \\
\text { formación en } \\
\text { su contexto }\end{array}$ & 16.597 & 20.746 & 12.448 & 12.015 & 38.174 \\
\hline
\end{tabular}

TABLA 5. Actitudes hacia la formación inicial en TIC de los participantes de la muestra en \%.

\begin{tabular}{|l|c|c|c|c|c|}
\hline & $\begin{array}{c}\text { Totalmente de } \\
\text { acuerdo }\end{array}$ & $\begin{array}{c}\text { De } \\
\text { acuerdo }\end{array}$ & Neutro & $\begin{array}{c}\text { En } \\
\text { desacuerdo }\end{array}$ & $\begin{array}{c}\text { Totalmente } \\
\text { en desacuerdo }\end{array}$ \\
\hline $\begin{array}{l}\text { Formación } \\
\text { Inicial }\end{array}$ & 0 & 4.151 & 23.746 & 41.908 & 30.195 \\
\hline $\begin{array}{l}\text { suficiente } \\
\text { Formación } \\
\text { Inicial } \\
\text { Instrumentalista }\end{array}$ & 25.311 & 20.896 & 12.597 & 19.599 & 13.597 \\
\hline $\begin{array}{l}\text { Mayor no s } \\
\text { de asignatura }\end{array}$ & 35.609 & 1.195 & 12.448 & 12.450 & 8.298 \\
\hline
\end{tabular}


TABLA 6. Actitudes hacia formación permanente en TIC de los participantes de la muestra en $\%$.

\begin{tabular}{|l|c|c|c|c|c|}
\hline & $\begin{array}{l}\text { Totalmente de } \\
\text { acuerdo }\end{array}$ & $\begin{array}{c}\text { De } \\
\text { acuerdo }\end{array}$ & Neutro & $\begin{array}{c}\text { En } \\
\text { desacuerdo }\end{array}$ & $\begin{array}{c}\text { Totalmente } \\
\text { en desacuerdo }\end{array}$ \\
\hline $\begin{array}{l}\text { El elevado } \\
\text { coste de } \\
\text { Formación } \\
\text { en NTIC }\end{array}$ & 21.597 & 18.298 & 51.792 & 4.659 & 3.654 \\
\hline $\begin{array}{l}\text { Donde estudio } \\
\text { o trabajo hay } \\
\text { ofertas de } \\
\text { Formación } \\
\text { en NTIC }\end{array}$ & 21.161 & 29.045 & 16.600 & 20.746 & 12.448 \\
\hline $\begin{array}{l}\text { He realizado } \\
\text { cursos, jornadas... }\end{array}$ & 8.298 & 33.195 & 24.896 & 29.045 & 4.566 \\
\hline $\begin{array}{l}\text { Falta de } \\
\text { Tiempo para } \\
\text { la formación } \\
\text { en NTIC }\end{array}$ & 30.459 & 23.896 & 8.300 & 25.897 & 11.448 \\
\hline $\begin{array}{l}\text { Cursos } \\
\text { demasiado } \\
\text { instrumental } \\
\text { en NTIC }\end{array}$ & 24.331 & 25.896 & 13.599 & 18.577 & 17.597 \\
\hline
\end{tabular}

\section{Bibliografía}

ALLPORT, G.W. (1935). Attitudes. En C. Murchison (dir.). A handbook of social psychology. Worcerter: Clark University Press.

ALLPORT, G.W. (1977). Psicología de la personalidad. Buenos Aires: Piados.

ALONSO, C. y GALLEGO, D., (1995). Formación del profesor en Tecnología Educativa. En D. Gallego y Otros. Integración curricular de los recursos tecnológicos. Barcelona: Oikos-Tau. pp: 31-64.

ALPISTE, F.; BRIGOS, M. Y MONGUET, J. M. (1993). Aplicaciones Multimedia. Presente y futuro. Barcelona: Técnicas Rede.

ANDERSON, L.W. (1981). Assessing Affective Characteristics in the Schools. Allyn and Bacon. Boston: Massachusetts.

AREA MOREIRA, M. (1991). Los medios, los profesores y el currículo. Barcelona: Sendai. 
AZNAR DÍAZ, I.; HINOJO LUCENA, F.J. y FERNÁNDEZ MARTÍN, F.D. (2001). Nuevas Tecnologías y Desarrollo Sociopersonal. Un Enfoque Educativo. Granada: Método Ediciones.

BALLESTA, J. (1996). La formación del profesorado en nuevas tecnologías aplicadas a la educación. En J. Salinas y Otros (Coords). Edutec95. Redes de comunicación, redes de aprendizaje. Palma de Mallorca: Universidad de las Islas Baleares. pp: 435-447.

BARTOLOME, A. R. (1989). Nuevas Tecnologías y Enseñanza. Barcelona: GRAO-ICE de la Universidad de Barcelona.

BLAZQUEZ, F. (1994). Propósitos formativos de las nuevas tecnologías de la información en la formación de maestros. En F. Blazquez, J. Cabero y F. Loscertales (Coords). Nuevas Tecnologías de la Información y Comunicación para la Educación. Sevilla: Alfar. Pp: 257-268.

BRUCE, M. G. Y OTROS (1991). Developing a Global Perspective: Strategies for Teacher Education Programas, Journal of Teacher Education, 42 (1). pp. 21-27.

BUELA CASAL, G. y SIERRA, J.C. (1997). Manual de Evaluación Psicológica. Fundamentos, técnicas y aplicaciones. Madrid: Siglo XXI.

CEBRIAN DE LA SERNA, M., (1996). Una nueva necesidad, una nueva asignatura. En J. Salinas y Otros (Coords). Redes de comunicación, redes de aprendizaje Edutec95. Palma de Mallorca: Universidad de las Islas Baleares. pp: 471-476.

COLOM, A.; SUREDA, J. Y SALINAS, J. (1988). Tecnología y medios educativos. Madrid: Cincel.

DE PABLOS, J. (1996). Tecnología y Educación. Barcelona: Cedecs.

DE PABLOS, J. Y GORTARI, C. (Eds.) (1992). Las Nuevas Tecnologías de la Información en la Educación. Sevilla: Alfar.

DEL MORAL PÉREZ, M.E. (1995). Los recursos tecnológicos en el ámbito escolar. Madrid: Instituto de Tecnología Educativa de CECE.

DELGADO, L. Y SAENZ BARRIO, O. (1993). Organización Escolar. Una perspectiva ecológica. Alcoy: Marfil.

ESCUDERO, J. M. (1995). Tecnología e innovación educativa, Bordón, 47 (2). pp.161174.

ESCUDERO, J. M. Y LÓPEZ YÁÑEZ, J. (1992). Los desafios de las reformas escolares. Sevilla: GID.

FISHBEIEN, M. Y AJZEN, I. (1975). Belief, Attitude, Intention, and Behavior: An Introduction to Theory an research. Massachussets: Addison-Wesley Reading.

FUENTES ESPARREL, J. A. (2002). Tendencias en la formación del profesorado y barreras que obstaculizan la integración curricular de los Medios y Tecnologías de la Información y la Comunicación. En M. Lorenzo, J. A. Ortega et al. (Coords). Liderazgo educativo y escuela rural. Granada: Grupo Editorial Universitario. pp: 615640.

GALLEGO, D. J.; ALONSO, C. M. Y CANTON, I. (coords.) (1996). Integración curricular de los recursos tecnológicos. Barcelona: Oikos-tau. 
HINOJO LUCENA, F. J. y FERNÁNDEZ MARTíN, F. D. (2002). Diseño de escalas de actitudes para la formación del profesorado en Tecnologías, Revista lberoamericana de Comunicación y Educación, no 19. pp: 120-125.

LIEBERMAN, A. (Ed.) (1988). Building a professional culture in schools. New York: Tacher College Press.

LIPOVETSKY Y GILLES (1986). La era del vacío. Ensayos sobre el individualismo contemporáneo. Barcelona: Anagrama.

LISTON, D. P. Y ZEICHNER, K. M. (1993). Formación del profesorado y condiciones sociales de la escolarización. Madrid: Morata.

LOPEZ HERRERÍAS, J. A. (1989). El profesor educador: persona y tecnólogo. Madrid: Cincel.

LYNCH, J. (1989). Multicultural Education in a Global Society. London: The Falmer Press.

LYOTARD, J. (1979). La condition postmoderne. París: le Minuit.

M.E.C. (1969). La educación en España. Bases para una política educativa. Madrid: M.E.C.

MARRERO, J. (1988). Teorías implícitas del profesorado y curriculum, Cuadernos de Pedagogía, 197. pp. 66-69.

MARTÍNEZ SÁNCHEZ, F. (1990). La educación ante las nuevas tecnologías de la comunicación. Configuración de videos didácticos, Anales de Pedagogía, 8. pp. 159-180.

MASTERMAN, L. (1983). La educación en materia de comunicación: problemas teóricos y posibilidades concretas, Perspectivas, Vol XIII, 2.

MEDINA RIVILLA, A. (1989). La formación del profesorado en una sociedad tecnológica. Madrid: Cincel.

MONTERO, A. (1994). Pensamiento y creencias. Sevilla: C. de Educación de la Junta de Andalucía.

MUÑOZ REPISO Y OTROS (Edit.) (1992). Educación y valores en España. Madrid: CIDE-MEC.

OLSON, J.M. y ZANNA, M.P. (1993). Attitudes and attitude change, Annual Review Psychology, 44, Pp: 117-154.

RISPA, R. (1985). Nuevas Tecnologías en la vida cultural española. Madrid: FundesCO.

RODRIGUEZ, J. L. Y SAENZ, O. (1995). Tecnología Educativa. Nuevas tecnologías aplicadas a la Educación. Madrid: Marfil.

SANCHO, J. M. (coord.) (1994): Para una tecnología educativa. Barcelona: Horsori.

SCHÖN, D. A. (1987). Educating the reflective Practitioner. Barcelona: Paidós.

SUMMERS, G. F. (1976). Medición de actitudes. México: Trillas.

TEJEDOR, F.J. Y GARCIA-VALCARCEL, A. (coord.) (1996). Perspectivas de las Nuevas Tecnologías en la Educación. Madrid: Narcea.

TRIANDIS, H. C. (1974). Actitudes y cambios de actitudes. Barcelona: Toray. 
FRANCISCO D. FERNÁNDEZ MARTÍN, FRANCISCO JAVIER HINOJO LUCENA, INMACULADA AZNAR DÍAZ

VILLAR, L. M. (1996). La formación permanente del profesorado en el Nuevo Sistema Educativo de España. Barcelona: Oikos-Tau.

ZEICHNER, K.M. Y GORE, J. M. (1990). Teacher socialization, en Houston (Ed.): Handbook of Reserach on Teacher Education, N. York, McMillan, pp. 329-348. 